\title{
Two Important Features of the 156 Deadliest Tropical Cyclones (1851-1996) in the North Atlantic
}

\author{
Bomin Chen1, Jiqing Tan²*, Huiyi Fan² \\ ${ }^{1}$ Shanghai Climate Center, Shanghai, China \\ ${ }^{2}$ Earth Science School, Zhejiang University, Hangzhou, China \\ Email: *tanjiqing@126.com
}

How to cite this paper: Chen, B.M., Tan, J.Q. and Fan, H.Y. (2019) Two Important Features of the 156 Deadliest Tropical Cyclones (1851-1996) in the North Atlantic. Atmospheric and Climate Sciences, 9, 75-83.

https://doi.org/10.4236/acs.2019.91005

Received: September 25, 2018

Accepted: December 11, 2018

Published: December 14, 2018

Copyright (c) 2019 by authors and Scientific Research Publishing Inc. This work is licensed under the Creative Commons Attribution International License (CC BY 4.0).

http://creativecommons.org/licenses/by/4.0/

\section{(c) (i) Open Access}

\begin{abstract}
Damages and deaths due to a tropical cyclone (TC) can easily occur at three places belong to a TC: the small ring area (SRA) of maximum sustaining wind around TC eye, the large ring area (LRA) of the rotating wind field around SRA and the skin air belt (SKAB) around LRA. However, weather forecasters only use the intensity of the SRA of a TC as the proxy to gauge the whole TC intensity, which led to many "non-major" TCs proved to be "deadly" TCs. This is called as "over-generalization problem" (OGP). Here we introduce a new way to measure the intensity of a TC. After investigating 156 deadly TCs in North Atlantic, we found an important feature: 83.3\% of the top 156 deadliest TCs usually made landfalls along "five major tracks". Furthermore, we found that the new intensity of those "deadliest" TCs kept almost unchanged with the increasing of time intervals at earlier stage from the genesis points, whereas the new intensity of those "safe" TCs increased in a line with much steeper slope. Using these two features, weather forecasters can have two more options to identify those "non-major" and "deadly" TCs by SSHS in future.
\end{abstract}

\section{Keywords}

Over-Generalization Problem, Tropical Cyclone, Intensity

\section{Introduction}

Pioneer researchers considered that the pathway patterns of tropical cyclones (TCs) are very important information in the study of the TC formation mechanism [1]-[11]. Specifically, the formation of TCs can be better understood by us- 
ing a track classification method to survey historical TCs [12] [13] [14]. Despite many years of effort by scientists [15]-[22], a theory capable of completely explaining the physical mechanism of TC formation has not yet emerged. As such, it is very important to find out the deficiency of present TC intensity evaluating system.

In the present TC intensity evaluating systems in the world, the maximum sustaining winds are the proxy to measure TC intensity, and the Saffir-Simpson Hurricane Scale (SSHS) is the leading TC intensity method. In SSHS, a TC below category 3 is called a "non-major" TC, which means that the TC is a "weak" TC on their intensity, and a TC above category 3 is called a "major" TC, which means that the TC is a "strong" TC. Because only about $10 \%$ of TCs were "deadly" TCs, common people often choose to stay home or to leave for a safe place depending on "weak" or "strong" intensity of TCs. Generally, the information of "weak" or "strong" intensity for a TC is according to the information of "non-major" and "major" TCs in TC warnings by weather forecasters. However, this usual policy was not always reliable. For example, Hurricane Gordon, which occurred from November 8 to 21, 1994, was classified as a "non-major" TC according to SSHS, but Hurricane Gordon was the 16th deadliest TC over North Atlantic the period from 1851 to 1996 . Some scholars had criticized the SSHS as being too simplistic because of two substantial limitations: its duration problem and its failure to take the physical size of TCs into accounts [23] [24] [25]. In our opinion, the bad performance of TC warnings by weather forecasters was not due to the deficiency of SSHS, but due to that there is an over-generalization problem (OGP) existing in the present TC intensity evaluating systems.

In meteorology, the coverage area of a weather system is also one of the quantities to gauge the intensity of a weather system. In this paper, we collectively call all the three areas as "most direct impact area" (MDIA), the calculating scheme can be seen in the previous literature [26] [27]. Here we use the instant MDIA, which is the coverage area when a TC moves from one place to another at the intervals of six hours, as the intensity of a TC. Noticing that most deadly TCs moved very slowly, thus, we deduce that the 156 deadliest TCs might have less amount of instant MDIA, which we might find some important information to distinguish deadly TCs from those "safe" TCs. On the other hand, common people need to know where the safe places are when a TC is coming; thus, it is another target to seek for what kinds of tracks, which the 156 deadly TCs preferred to move.

\section{Methods}

\subsection{Calculation Method of Instant MDIA}

$$
I_{m \text { dia }}=\frac{1}{N} \sum \frac{S_{i, j}}{T}
$$

where $T$ is the interval time (six hours), $S_{i, j}$ is the area of 1 unit area, and $N$ is the total number of grid points in the instant MDIA, which is calculated with 
formula (2)

$$
S_{i, j}=0.01 * C^{2} * R^{2} * \operatorname{COS} \phi
$$

where $C$ is $0.01745329, \mathrm{R}$ is the radius of the earth, and $\phi$ is the latitude.

The derivation of (1) is as follows:

$$
\begin{aligned}
S_{i, j} & =d x * d y=(0.1 * \pi / 180 * R) *(0.1 * \pi / 180 * R * \operatorname{COS} \phi) \\
& =0.01 *(\pi / 180)^{2} * R^{2} \operatorname{COS} \phi=0.01 * C^{2} R^{2} \operatorname{COS} \phi
\end{aligned}
$$

where $C \equiv \pi / 180 \approx 0.01745329$.

\subsection{The Method to Find the Five Groups of TCs}

We use two cross lines $\mathrm{X}$ and line $\mathrm{Y}$ to divide the North Atlantic Ocean basin. The equations for $\mathrm{X}$ line is in the formula (4) and (5)

$$
y=0.4172 x+59.298
$$

Due to the mathematical relation that the product of the slope of the $X$ axis and the slop of the $Y$ axis must equal -1, we can obtain the slope and equation for the $Y$ axis with the following formula:

$$
y=-2.3969 x-110.1915
$$

where $x$ is degrees of longitude and $y$ is degrees of latitude.

\section{Results}

In Table 1, the first column lists the 0 - 5 categories according to SSHS; the second column lists the number of TCs which caused deaths; the third column lists the toll of deaths caused by TCs belong to this category. We can see that the maximum number of TCs causing deaths is category 3 , there were 46 deadly TCs during the periodic from 1851 to 1996, which caused 18,340 people to die; then the number order from the second maximum number to minimum number is: category 4, category 2, category 1 , category 5 and category 0 , respectively, their death numbers are: 45,176, 14,614, 7896, 7110 and 1092. Summing the numbers of deaths belong to category 2, 1 and 0 , we obtained 23,602 deaths by "non-major" TCs according to SSHS; Summing the numbers of TCs belong to category 2, 1 and 0, we obtained 55 "non-major" TCs according to SSHS, which is about " $35.2 \%$ " of 156 deadliest TCs during the period from 1851 to 1996. The reason for this phenomenon is due to that damages and deaths due to a tropical cyclone (TC) can easily occur at three places belong to a TC: the small ring area (SRA) of maximum sustaining wind around TC eye, the large ring area (LRA) of the rotating wind field around SRA and the skin air belt (SKAB) around LRA (see Figure 1). However, in SSHS, the intensity of the SRA of a TC is used to gauge the whole TC intensity, which led to that many "non-major" TCs proved to be "deadly" TCs. This is called as "over-generalization problem" (OGP).

In Figure 2, we illustrate the arithmetic sum of the instant MDIA of all 156 deadly TCs on a geographical grid with a fixed 0.1-degree resolution; we obtained the geographic distribution of instant MDIA, which can show all the 
Table 1. Death toll of the 156 top deadliest tropical cyclones in North Atlantic.

\begin{tabular}{ccc}
\hline Categories & Numbers & Total Death Numbers \\
\hline 0 & 13 & 1092 \\
1 & 20 & 7896 \\
2 & 22 & 14614 \\
3 & 46 & 18340 \\
4 & 41 & 45176 \\
5 & 14 & 7110 \\
\hline
\end{tabular}

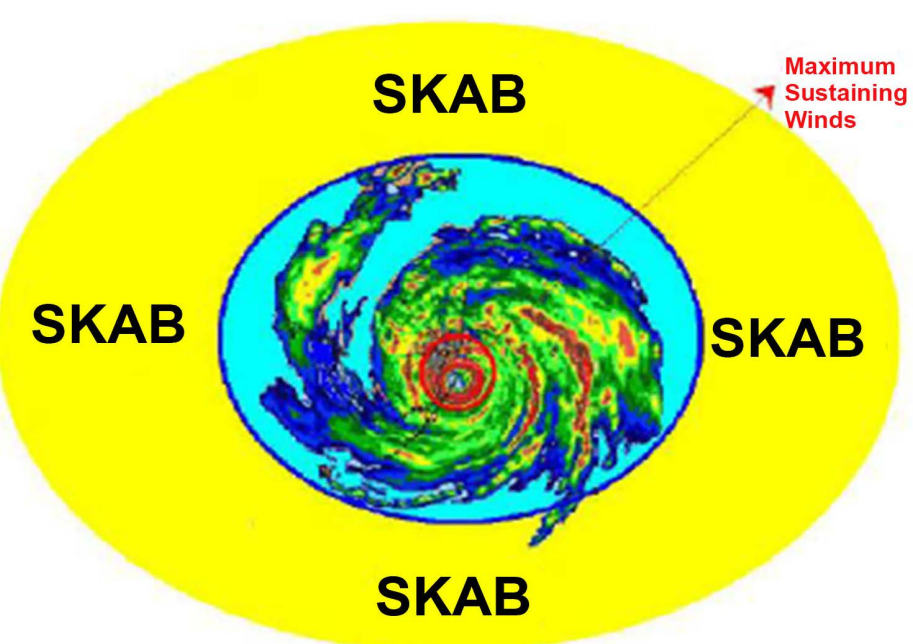

Figure 1. The new intensity evaluating system. Notes: 1) The small ring area marked in red is called small ring area (SRA). The SSHS use the maximum sustaining winds to define the intensity of a TC. 2) The big ring area marked in blue is called large ring area (LRA). 3) The skin air belt refers to those air belts around LRA. This area is special place due to it receiving kinetic energy when a TC moves.

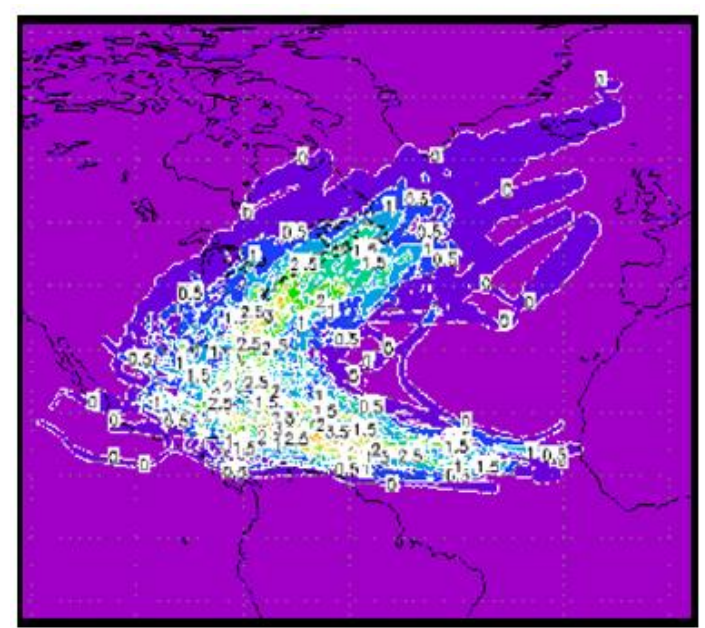

Figure 2. The geographic distribution of instant MDIA of 156 deadly TCs (1851-1996). Notes: the unit is $1 . * 10^{9} \mathrm{M}^{2} / \mathrm{S}$. 
tracks of 156 top deadliest TCs. We can see that the geometry of all the tracks of 156 top deadliest TCs resembles the letter "L".

In Figure 3, we illustrate the geographic distribution of the instant MDIA of five groups of TCs and we also give a pathway line of a typical cases' for each of five groups of TCs.

In Figure 4, it shows the average values of instant MDIA with time for both "safer" 1282 TCs and 156 deadliest TCs according to the order numbers of different time from genesis points to dissipation points. We could see that the slope of the instant MDIA curve line for the 1282 "safer" TCs is steeper than that of 156 deadly TCs, while the curve line for the 156 top deadliest TCs keeps almost unchanged if the lifespan of a TC is shorter than 10 days; it increases upward to a peak and decrease downward to a valley for four times during the lifespan from 11 days to 18 days, but the amplitude is much less than that in the curve line for the 1282 "safer" TCs.

\section{Discussion}

Due to only the intensity of SRA of a TC is the proxy to measure TC intensity in SSHS used by weather forecasters to submit TC warnings, and due to the intensities in LRA and SKAB remain undefined in the TC evaluating systems in the world; the OGP problem would occur to leads to that many "non-major" TCs proved to be deadly TCs. Table 1 shows the bad performance of the present TC intensity evaluating systems over North Atlantic. 23,602 deaths had been caused by "non-major" deadly TCs in SSHS, which supports our view on OGP in present TC intensity evaluating system in the world. Other scholars had blamed the defects of SSHS as the reason leading to the bad performance, but we consider that the OGP in present TC intensity evaluating is the real reason.

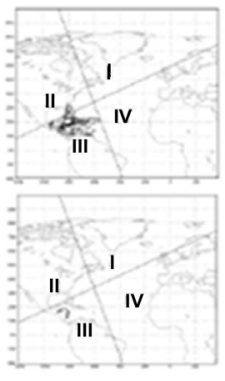

(a)

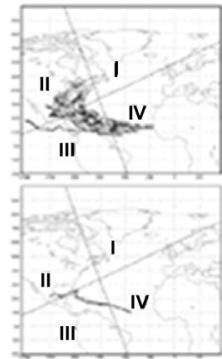

(b)

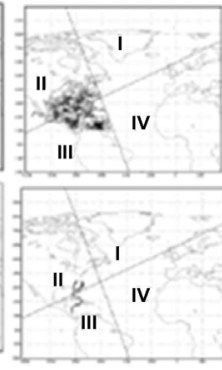

(c)

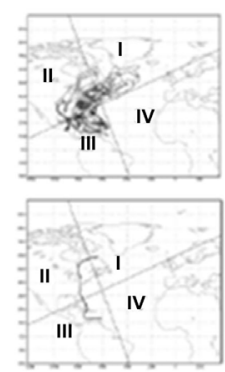

(d)

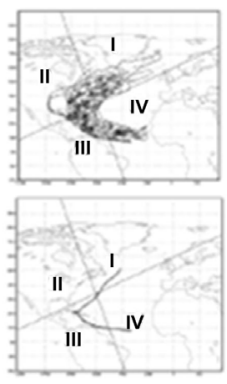

(e)

Figure 3. The geographic distribution of instant MDIA of Five groups of deadly TCs (1851-1996) (the unit is $1 . * 10^{9} \mathrm{M}^{2} / \mathrm{S}$ ). Note: (a) TCs' genesis and dissipation points are all located at in region III (a typical case: Hurricane Hattie in 1961). (b) TCs' genesis points all located at in region IV, and TCs' dissipation points all located at in region II (a typical case: Hurricane Inez in 1966). (c) TCs' genesis points all located at in region III, and TCs' dissipation points all located at in region II (a typical case: Hurricane Gordon in 1994). (d) TCs' genesis points all located at in region III, and TCs' dissipation points all located at in region I (a typical case: Hurricane Hazel in 1954). (e) TCs' genesis points all located at in region IV, and TCs' dissipation points all located at in region I (a typical case: Hurricane Flora in 1963). 


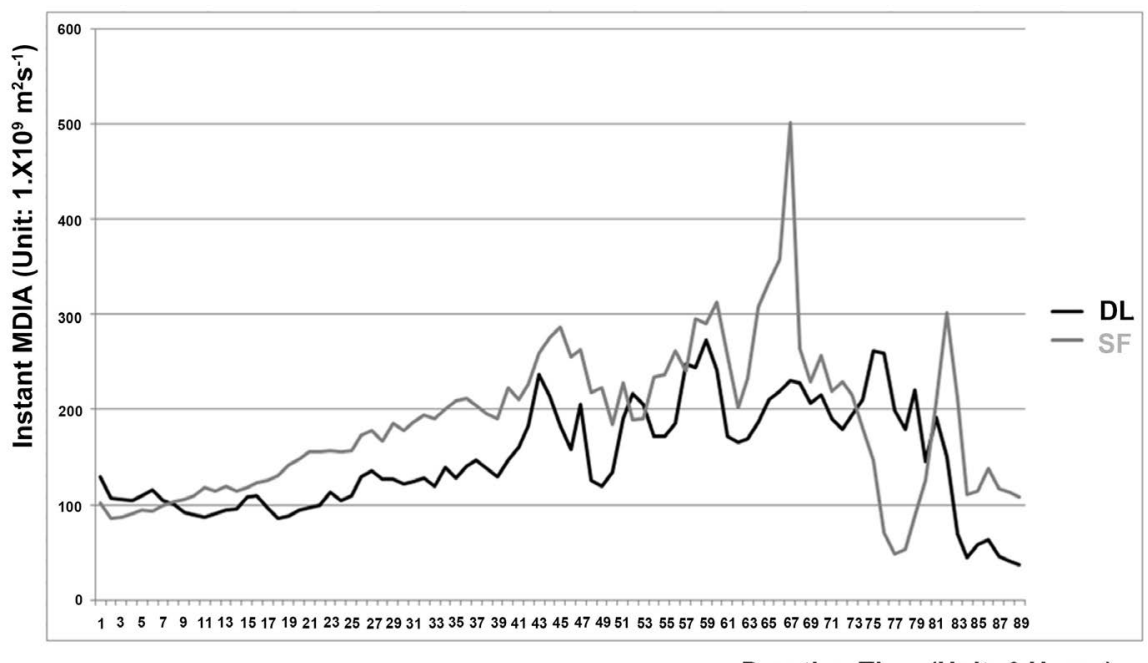

Duration Time (Unit: 6 Hours)

Figure 4. The average values of instant MDIA in the time order from genesis point to dissipation points for two groups of TCs: 1282 "safe" TCs and 156 deadliest TCs (1851-1996). Note: DL: the black line stands for 156 deadliest TCs; SF: the grey line stands for 1282 "safe" TCs.

One of the direct ways to overcome OGP is to establish two TC intensity evaluating sub-systems: the sub-system to evaluate the TC intensity of LRA and the sub-system to evaluate the TC intensity of SKAB. Here in this paper, we try one of the indirect ways: to use instant MDIA to gauge TC intensity. As you may know, the coverage area of a weather system is another attributes to characterize the intensity in meteorology, the instant MDIA which is calculated based on the best-track position data set, cover all the three areas the direct way to other scholars have confirmed the existence of OGP in present TC intensity evaluating systems, In this paper, it suggests that we can take some measures to make some compensation for the deficiency of OGP.

First, we have found five major tracks of 156 deadliest TCs based on the geographic distribution data of instant MDIA of 131 TCs (see Figure 3), it takes 83.3\% of the 156 deadliest TCs. Due to $35.2 \%$ of 156 deadliest TCs were "non-major" TCs, the five major tracks can be used as a more opportunity to improve their forecast skill. For example, if a non-major TCs is expected to make a landfall according to SSHS; the weather forecasters should check the coming non-major TC to see if its track belongs to any of the five track patterns or not, if the answer is "yes", he would have an option to re-evaluate the coming non-major TC as a deadly TC.

Second, due to the coverage area of a weather system can be used to express the intensity of a weather system in meteorology, instant MDIA is thus to be used to gauge the intensity of a TC. For example, if two TCs are non-major TCs by SSHS, which means that the two TCs are "weak" in SRA of a TC, but their intensities in LRA and SKAB remain unknown. Many non-major TCs proved to be very strong might be due to the intensities of other two regions were "very strong". Using the instant MDIA as the proxy to gauge the TC intensity, and we 
found the instant MDIA intensity of 156 deadly TCs keep unchanged or slightly changed at their earlier stage from their genesis points, whereas the instant MDIA intensity of those 1282 "safe" TCs increase remarkably. Due to the instant MDIA intensity refers to the coverage of the area from one position to another at six hour intervals, therefore, the larger the instant MDIA of a TC is, the faster the TC moves. In Figure 3, it illustrates the average values of the instant MDIA at different interval times according to the order numbers from their genesis points to their dissipation points; we can see the average instant MDIA curve lines for two groups of TCs are quite different. Due to this feature occur in the earlier stage of the lifespan of a TC, it can be used by weather forecasters to re-consider the intensities of the two TCs.

\section{Conclusion}

In this paper, we point out that there is an OGP existing in present TC intensity evaluation system; a statistic toll for 156 deadly TCs and new terms of SRA, LRA and SKAB has been used to explain that the view is correct. In order to overcome OGP, we have developed a new track classification method to identify the five kinds of deadly TC tracks, furthermore, an important feature of deadly TCs has been obtained. At last, we suggest two measures to compensate for the OGP.

\section{Acknowledgements}

This paper is supported by three projects: 1) National Natural Science Foundation of China (Grant NO.40875091), 2) Special Scientific Research Project for Public Interest (Grant NO. GYHY201306021), and 3) the National Key Research and Development Program of China "major natural disaster monitoring warning and prevention” (Grant No. 2017YFC1502301).

\section{Conflicts of Interest}

The authors declare no conflicts of interest regarding the publication of this paper.

\section{References}

[1] Palmen, E. (1948) On the Formation and Structure of Tropical Hurricanes. Geophysica, 3, 26-38.

[2] Riehl, H. (1948) On the Formation of Typhoons. Journal of Meteorology, 5, 254-264. https://doi.org/10.1175/1520-0469(1948)005<0247:OTFOT >2.0.CO;2

[3] Sawer, S. (1947) Notes on the Theory of Tropical Cyclones. Quarterly Journal of the Royal Meteorological Society, 73, 101-126. https://doi.org/10.1002/qj.49707331509

[4] Syono, S. (1953) On the Formation of Tropical Cyclone. Tellus, 5, 179-195. https://doi.org/10.3402/tellusa.v5i2.8571

[5] Kuo, H.L. (1965) On Formation and Intensification of Tropical Cyclones through Latent Heat Release by Cumulus Convection. Journal of the Atmospheric Sciences, 22, 40-63. https://doi.org/10.1175/1520-0469(1965)022<0040:OFAIOT >2.0.CO;2

[6] Yanai, M. (1964) Formation of Tropical Cyclones. Reviews of Geophysics, 2, 367-414. 
https://doi.org/10.1029/RG002i002p00367

[7] Ooyama, K. (1969) Numerical Simulation of the Life Cycle of Tropical Cyclones. Journal of the Atmospheric Sciences, 26, 3-40. https://doi.org/10.1175/1520-0469(1969)026<0003:NSOTLC >2.0.CO;2

[8] Pederson, T.S. (1991) A Comparison of the Free Ride and CISK Assumption. Journal of the Atmospheric Sciences, 48, 1813-1821. https://doi.org/10.1175/1520-0469(1991)048<1813:ACOTFR>2.0.CO;2

[9] Rotunno, R. and Emanuel, K. (1987) An Air-Sea Interaction Theory for Tropical Cyclones. Part II: Evolutionary Study Using a Non-Hydrostatic Axisymmetric Model. Journal of the Atmospheric Sciences, 44, 542-561. https://doi.org/10.1175/1520-0469(1987)044<0542:AAITFT>2.0.CO;2

[10] Challa, M. and Pfeffer, R. (1990) Formation of Atlantic Hurricanes from Cloud Clusters and Depressions. Journal of the Atmospheric Sciences, 47, 909-927. https://doi.org/10.1175/1520-0469(1990)047<0909:FOAHFC>2.0.CO;2

[11] Bosart, L. and Bartlo, J. (1991) Tropical Storm Formation in a Baroclinic Environment. Monthly Weather Review, 119, 1979-2013. https://doi.org/10.1175/1520-0493(1991)119<1979:TSFIAB>2.0.CO;2

[12] Chu, C.C. (1918) Some New Facts about the Centers of Typhoons. Monthly Weather Review, 46, 417-419. https://doi.org/10.1175/1520-0493(1918)46<417:SNFATC >2.0.CO;2

[13] Chu, C.C. (1924) A New Classification of Typhoons of the Far East. Monthly Weather Review, 52, 136-139. https://doi.org/10.1175/1520-0493(1924)52<570:ANCOTO>2.0.CO;2

[14] Chu, C.C. (1925) The Place of Origin and Recurvature of Typhoons. Monthly Weather Review, 53, 1-5. https://doi.org/10.1175/1520-0493(1925)53<1:TPOOAR>2.0.CO;2

[15] Haque, S.M.A. (1952) Initial of Cyclonic Circulation in a Vertically Unstable Stagnant Air Mass. Quarterly Journal of the Royal Meteorological Society, 78, 394-406. https://doi.org/10.1002/qj.49707833710

[16] Lily, D.K. (1960) On the Theory of Disturbances in a Conditionally Unstable Atmosphere. Monthly Weather Review, 88, 1-17. https://doi.org/10.1175/1520-0493(1960)088<0001:OTTODI>2.0.CO;2

[17] Yanai, M. (1961) A Detailed Analysis of Typhoon Formation. Journal of the Meteorological Society of Japan, 39, 187-213. https://doi.org/10.2151/jmsj1923.39.4_187

[18] Charney, J. and Eliassen, A. (1964) On the Growth of the Hurricane Depression. Journal of the Atmospheric Sciences, 21, 68-75. https://doi.org/10.1175/1520-0469(1964)021<0068:OTGOTH>2.0.CO;2

[19] Pederson, T.S. and Rasmussen, E. (1985) On the Cut-Off Problem in Linear CISK Models. Tellus A, 37, 394-402. https://doi.org/10.1111/j.1600-0870.1985.tb00438.x

[20] Emanuel, K. (1991) The Finite-Amplitude Nature of Tropical Cyclogensis. Journal of the Atmospheric Sciences, 46, 3431-3456. https://doi.org/10.1175/1520-0469(1989)046<3431:TFANOT>2.0.CO;2

[21] Montgomery, M.T. and Farrell, B.F. (1993) Tropical Cyclone Formation. Journal of the Atmospheric Sciences, 50, 285-310. https://doi.org/10.1175/1520-0469(1993)050<0285:TCF $>2.0 . C O ; 2$

[22] Smith, R.K. (1997) On the Theory of CISK. Quarterly Journal of the Royal Meteorological Society, 123, 407-418. https://doi.org/10.1002/qj.49712353808 
[23] Kantha, L. (2006) Time to Replace the Saffir-Simpson Hurricane Scale? Eos, 87, 3-6. https://doi.org/10.1029/2006EO010003

[24] Powell, M.D. and Reinhold, T.A. (2007) Tropical Cyclone Destructive Potential by Integrated Kinetic Energy. Bulletin of the American Meteorological Society, 88, 513-526. https://doi.org/10.1175/BAMS-88-4-513

[25] Kantha, L. (2008) Tropical Cyclone Destructive Potential by Integrated Kinetic Energy. Bulletin of the American Meteorological Society, 89, 219-221.

[26] Tan, J.Q. (2013) A New Classification of Typhoons over Western North Pacific. Remote Sensing Science, 1, 21-26.

[27] Tan, J.Q., Li, H.Q. and Zheng, Z.X. (2011) New Climate Diagnostic Method of Activity of Typhoons over the Northwestern Pacific Ocean. Applied Mechanics and Materials, 71-78, 3118-3122. 